\title{
Effect of Extraction Time on the Rheological Properties of Sericin Solutions and Gels
}

\author{
Young Jin Yoo and In Chul Um* \\ Department of Bio-fibers and Materials Science, Kyungpook National University, Daegu 702-701 Korea
}

\section{Abstract}

Recently, silk sericin has attracted the attention of researchers owing to its useful properties as a biomaterial including 1) good wound healing and cell activities, 2) fast gelation character, and 3) high water retention property. In the present study, silk sericin was prepared using different extraction times in hot water and the effect of extraction time on the rheological properties of sericin solutions and gels was examined. It was found that the production yield of sericin increased with extraction time. The shear viscosity of sericin solutions and gels decreased with increasing extraction time due to a decrease in sericin molecular weight. When the sericin solution transformed to a gel, the viscosity increased and the shear thinning behavior was more evident. In addition, the shear stress measurements indicated that the slip between the sericin samples and the measuring plate of the rheometer was increased by the gelation of sericin. The compression strength of sericin gel could be increased remarkably (by more than 100 fold) by preparation using the freezing and thawing method.

(c) 2013 The Korean Society of Sericultural Sciences

Int. J. Indust. Entomol. 27(1), 180-184 (2013)

Received : 24 Aug 2013

Revised : 16 Sep 2013

Accepted : 17 Sep 2013

Keywords:

Sericin,

Solution,

Gel,

Extraction time,

Rheological properties

\section{Introduction}

Silk has been used as an excellent textile material for a long time. Although silk is composed of both fibroin and sericin, the sericin is usually removed by a degumming process to improve the luster and handling of silk fibers. Therefore, sericin has been considered a redundant material for a long time. However, recent extensive studies on silk sericin have reported on the suitability of sericin as a biomaterial. For instance, sericin showed a high water retention making it possible to be used as a water retention agent in cosmetic products. Sericin showed good wound- healing performance (Nagai et al., 2009) and good cell activities (Tsubouchi et al., 2005). Also, it was reported that sericin showed blood glucose and cholesterol lowering properties when ingested (Limpeanchob et al., 2010; Seo et al., 2011). These unique properties of sericin have attracted the attention of researchers for cosmetic, functional health food, and biomedical applications.

Sericin can be extracted using hot water because it is hydrophilic. However, it does not dissolve immediately in hot water and needs considerable time for the extraction. In addition, hydrolytic molecular degradation of sericin takes place during the hot-water extraction process. Therefore, as the extraction

\section{${ }^{*}$ Corresponding author.}

In Chul Um

Department of Bio-fibers and Materials Science, Kyungpook National University, Daegu 702-701, Korea

Tel: +82-53-950-7757 / FAX: +82-53-950-6744

E-mail: icum@knu.ac.kr 
time is increased, more sericin can be extracted, but at the same time, the sericin molecules become more degraded. Considering that many studies have been conducted to improve the mechanical properties of sericin and to extract high molecular weight (MW) sericin (Oh et al., 2007, 2011; Yun et al., 2013), it is clearly desirable to avoid the severe molecular degradation of silk sericin during the extraction process. That is, it is important to properly control the extraction time of sericin in hot water to simultaneously obtain both a high yield and a high MW.

Rheology has previously been used as an indicator to examine the structure of materials and to evaluate their processability for various applications including gelation (Yoo and Um, 2013), wet spinning (Cho et al., 2012) and electrospinning (Cho et al., 2012; Ko et al., 2013).

In this study, sericin was obtained using different extraction times in hot water and rheological measurements were performed on the resulting sericin solutions and gels to gain a better understanding of the effect of extraction time on the solution properties and gel character of sericin.

\section{Materials and Methods}

\section{Preparation}

Baekokjam silkworm cocoons were used for the silk sericin extraction. The preparation method of sericin has been introduced in a previous report (Jo et al., 2013). In brief, the silk cocoons were immersed at $100{ }^{\circ} \mathrm{C}$ for $30-120 \mathrm{~min}$. The liquor ratio was 1:25. After the hot water treatment, a sericin aqueous solution was obtained. The concentration of sericin aqueous solution was adjusted to $0.3 \%(\mathrm{w} / \mathrm{v})$ and stored at 5 ${ }^{\circ} \mathrm{C}$ for 3 days to prepare the sericin gel. Sericin gel was frozen at $-25{ }^{\circ} \mathrm{C}$ for a day and thawed at $25{ }^{\circ} \mathrm{C}$ for a day to fabricate the frozen and thawed sericin gels.

\section{Measurement and characterization}

The production yield of sericin was determined using the following equation:

Production yield $(\%)=$

$\frac{\text { Dry mass of silk sericin after extraction }}{\text { Dry mass of silk cocoons before extraction }} \times 100$
The dry mass of sericin and cocoons was determined using a moisture analyzer (XM60, Precesa, Switzerland).

Rheological measurement were performed on 1$)$ the $0.3 \%(\mathrm{w} /$ v) sericin aqueous solutions, 2) sericin gels prepared from the $0.3 \%$ sericin solution, and 3 ) frozen and thawed sericin gels. The steady-state flow of sericin solutions and gels were measured using a rheometer (MARS III, Hakke, Germany) using a $35 \mathrm{~mm}$ parallel-plate geometry at $25^{\circ} \mathrm{C}$. The compression strength of sericin gels was measured using the axial test function of the rheometer. The $35 \mathrm{~mm}$ plate of the rheometer compressed the gels with a speed of $0.12 \mathrm{~mm} / \mathrm{s}$.

\section{Results and Discussion}

\section{Production yield and amino acid composition}

Production yield is an important issue for the industrialization and mass production of silk sericin. Therefore, the production yield of sericin with different extraction times in hot water was determined and the results were shown in Fig. 1. With an extraction time of $30 \mathrm{~min}$, the production yield was $7.7 \%$. The yield increased with increasing extraction time and reached $11.4 \%$ at an extraction time of $120 \mathrm{~min}$. Considering that the sericin content of Baekokjam cocoons is about $26 \%$ (Ko et al., 2013), these values are not high. The yield can be increased further by increasing the extraction time or strengthening the treatment conditions including treatment temperature and pressure; however, in this case, molecular degradation of sericin can become a problem.

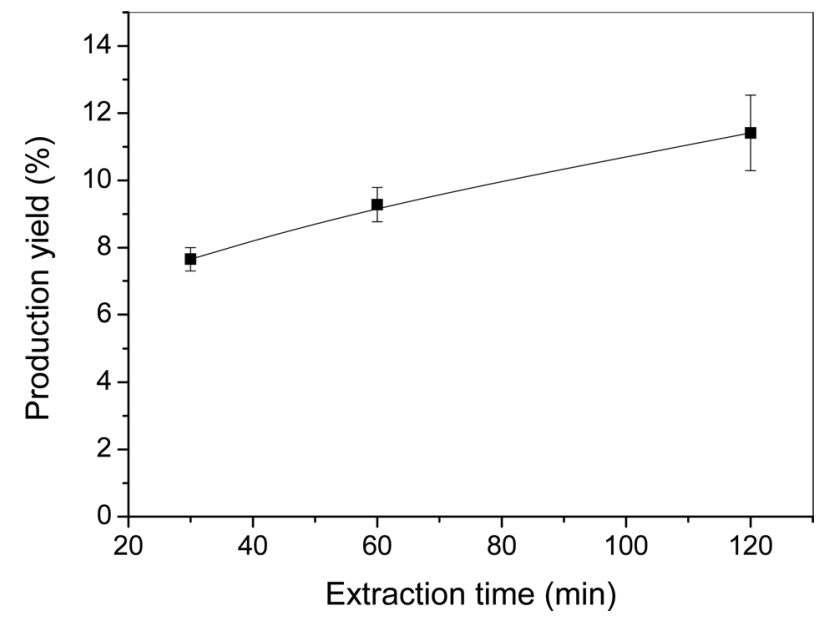

Fig. 1. Production yield of silk sericin with various extraction times. 


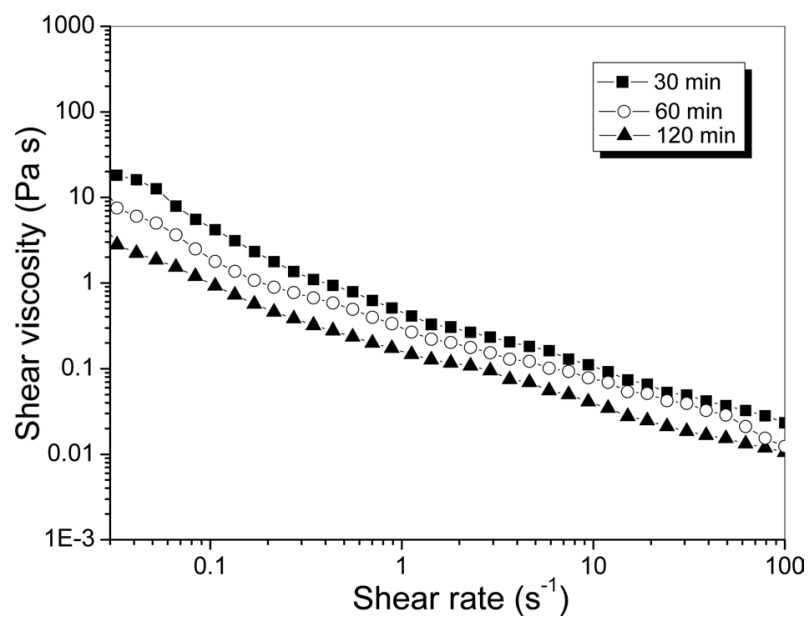

Fig. 2. Effect of extraction time on the steady-state flow of $0.3 \%$ (w/ v) silk sericin aqueous solutions.

\section{Rheological properties of sericin solutions and gels}

To examine the effect of extraction time on the rheological properties of sericin aqueous solution, the steady-state flow of $0.3 \%$ sericin aqueous solutions was measured using a rheometer and the results were presented in Fig. 2. A shear thinning behavior was observed in all sericin samples regardless of extraction time. As the extraction time was increased, the shear viscosity decreased. Fig. 3 shows the relationship between the shear stress and shear rate of $0.3 \%$ sericin aqueous solutions. For extraction times of $30 \mathrm{~min}$ and $60 \mathrm{~min}$, the shear stress decreased slightly at around $0.05 \mathrm{~s}^{-1}$ and then increased. Although an extraction time of $120 \mathrm{~min}$ did not show a decrease in shear stress, the shear stress at around $0.05 \mathrm{~s}^{-1}$ remained constant without increasing. When the shear stress at a certain shear rate decreases or remains constant, this indicates that a slip between the sericin aqueous solution and the rheometer measuring plate is occurring. This implies that the sericin aqueous solution is transforming into a gel state considering that 1) sericin aqueous solution becomes a gel easily and 2) a slip does not usually take place in the solution state.

The shear thinning behavior of the sericin solution samples might be due to the molecular entanglement or gelation of sericin. That is, neighboring molecules of sericin are easily aggregated and entangled resulting in shear thinning. Also, as can be seen in Fig. 3, gelation is taking place in all the sericin solution samples. In the case of a gel, the gel network is broken by the shear force, resulting in a decrease in viscosity. The

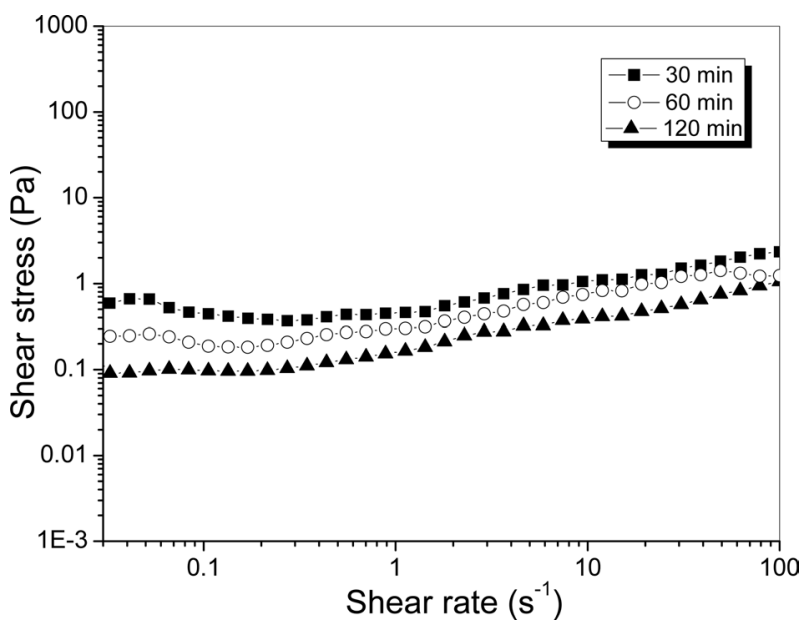

Fig. 3. Effect of extraction time on the relationship between shear stress and shear rate of $0.3 \%(\mathrm{w} / \mathrm{v})$ silk sericin aqueous solutions.

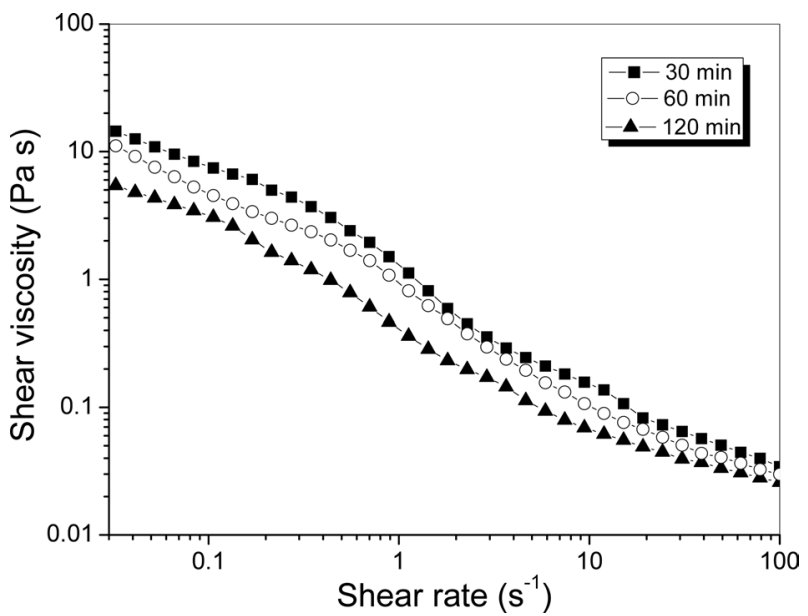

Fig. 4. Effect of extraction time on the steady-state flow of silk sericin gels.

decrease in shear viscosity with increasing extraction time is due to the hydrolytic molecular degradation of sericin in hot water.

Fig. 4 exhibits the steady-state flow of sericin gel produced by storing the sericin aqueous solutions at $4{ }^{\circ} \mathrm{C}$ for a day. Regardless of extraction time, all sericin gels showed a shear thinning behavior. Fig. 5 shows the shear stress of sericin gels as a function of shear rate. A 30 min extraction time resulted in a decrease in shear stress at two points: shear rates of $0.9 \mathrm{~s}^{-1}$ and $12 \mathrm{~s}^{-1}$. A 60 min extraction time showed a decrease in shear stress at $0.9 \mathrm{~s}^{-1}$. In the case of a $120 \mathrm{~min}$ extraction time, no decrease in shear stress was observed; however, a constant shear stress was shown at around $0.9 \mathrm{~s}^{-1}$. As mentioned above, a decrease or constant shear stress with increasing shear rate is due to a slip 


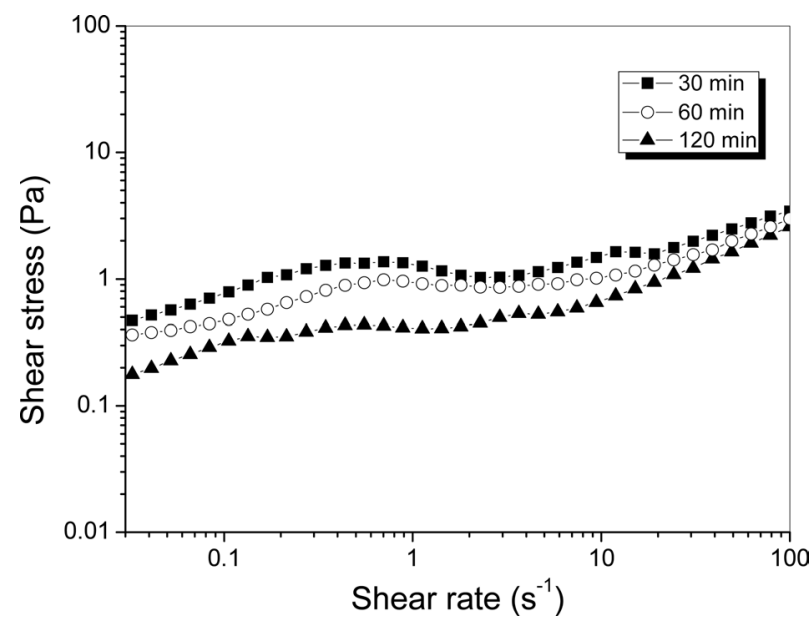

Fig. 5. Effect of extraction time on the relationship between shear stress and shear rate of silk sericin gels.

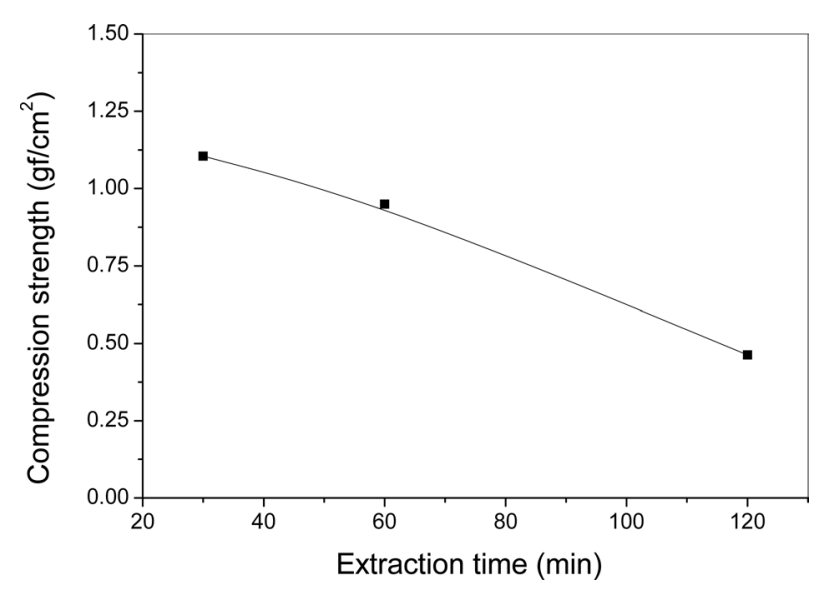

Fig. 6. Effect of extraction time on the compression strength of silk sericin gels.

between the sample and measuring plate of the rheometer. One different feature between the sericin solutions (Fig. 3) and gels (Fig. 5) is that the decrease in shear stress was more distinct in the sericin gel samples. This is because the slip between the plate and sample becomes more pronounced due to the stronger sericin gel structure indicated from Fig. 5. In other words, while the gelation of sericin was merely beginning in sericin solution samples (Fig. 3), the gelation was already finished in the sericin gel samples (Fig. 5). When the sericin gels were compared, the slip was more distinct for shorter extraction times. This is because the MW of sericin is higher at shorter extraction times, resulting in a stronger gel state and a more pronounced slip.

The reduction in shear viscosity of sericin gel with increasing extraction time implies that a stronger gel was formed at shorter

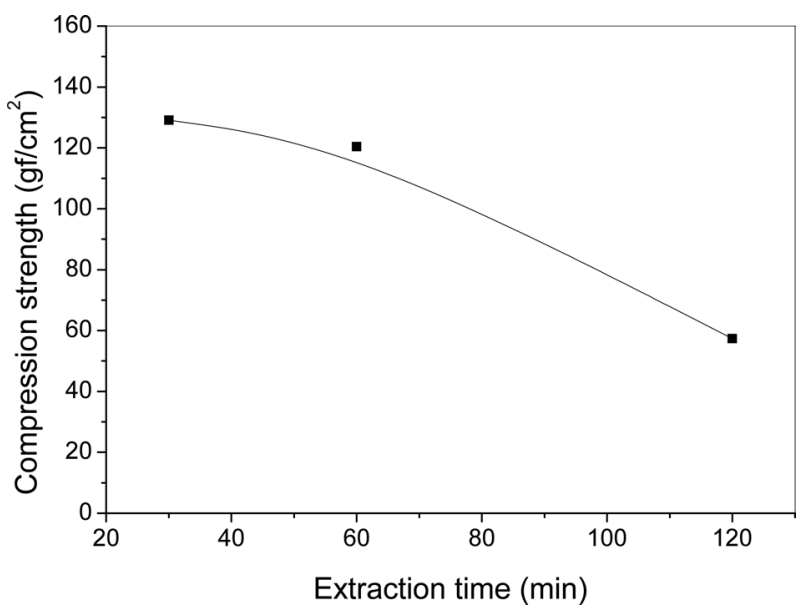

Fig. 7. Effect of extraction time on the compression strength of frozen-thawed silk sericin gels.

extraction times. This can also be understood considering that a higher MW sericin is produced at shorter extraction times and that a higher MW sericin results in a stronger gel. The shear thinning behavior of sericin gel became more distinct than that of sericin solution. A more pronounced difference between sericin solution and gel is that the decreasing slope of viscosity of sericin gel began to increase at around $0.7 \mathrm{~s}^{-1}$. This might be due to the breakdown of the gel network.

Fig. 6 exhibits the effect of extraction time on the compression strength of silk sericin gels. As observed, the compression strength of sericin gel decreased with increasing extraction time. This result reconfirms the earlier reasoning that a stronger sericin gel is formed by increasing the MW and decreasing the extraction time.

Fig. 7 shows the compression strength of silk sericin gels produced by a freezing and thawing method. The compression strength of frozen and thawed sericin gel showed a similar trend to that of normal sericin gel (Fig. 6). However, the compression strength of sericin was increased significantly (by more than 100 fold) by the freezing and thawing method, indicating that freezing and thawing is an effective fabrication method in improving the mechanical properties of sericin gel remarkably.

In the present study, silk sericin solutions and gels were prepared at different extraction times and the effect of extraction time on their rheological properties was examined. Rheological properties of sericin solutions and gels were determined as a function of 1) extraction time and 2) material state (solution or gel). Because the MW of sericin decreases with increasing extraction time, a longer extraction time resulted in a decrease in 
viscosity for both the solutions and gels. Both the viscosity and degree of shear thinning of sericin were increased by gelation. It was found that the freezing and thawing method was very effective at increasing the gel strength of sericin significantly.

\section{Acknowledgement}

This study was supported by the Basic Science Research Program through the National Research Foundation of Korea (NRF) funded by the Ministry of Education, Science and Technology (2012042016).

\section{References}

Cho HJ, Yoo HJ, Kim JW, Park YH, Bae DG, Um IC (2012) Effect of molecular weight and storage time on the wet- and electro-spinning of regenerated silk fibroin. Polym Degrad Stab 97, 1060-1066.

Jo YN, Bae DG, Um IC (2013) The effect of extraction conditions and film side on the molecular conformation of silk sericin film. Int J Indust Entomol 26, 113-118.

Ko JS, Yoon K, Ki CS, Kim HJ, Bae DG, Lee KH, Park YH, Um IC (2013) Effect of degumming condition on the solution properties and electrospinnablity of regenerated silk solution. Int J Biol Macromol $55,161-168$

Limpeanchob N, Trisat K, Duangjai A, Tiyaboonchai W, Pongcharoen S,
Sutheerawattananonda M (2010) Sericin reduces serum cholesterol in rats and cholesterol uptake into caco-2 cells. J Agric Food Chem 58, $12519-12522$.

Nagai N, Murao T, Ito Y, Okamoto N, Sasaki M (2009) Enhancing effects of sericin on corneal wound healing in Otsuka long-evans Tokushima fatty rats as a model of human type 2 Diabetes. Biol Pharm Bull 32, 1594-1599.

Oh HJ, Lee JY, Lee Y, Lee KH (2007) Enhanced mechanical property of silk sericin beads prepared from ethanol-precipitated sericin. Int J Indust Entomol 15, 171-174.

Oh HJ, Lee JY, Kim MK, Um IC, Lee KH (2011) Refining hot-water extracted silk sericin by ethanol-induced precipitation. Int J Biol Macromol 28, 32-37.

Seo C-W, Um IC, Rico CW, Kang MY (2011) Antihyperlipidemic and Body Fat-Lowering Effects of Silk Proteins with Different Fibroin/ Sericin Compositions in Mice Fed with High Fat Diet. J Agric Food Chem 59, 4192-4197.

Tsubouchi K, Igarashi Y, Takasu Y, Yamada H (2005) Sericin enhances attachment of cultured human skin fibroblasts. Biosci Biotechnol Biochem 69, 403-405.

Yoo YJ, Um IC (2013) Examination of thermo-gelation behavior of HPMC and HEMC aqueous solution using rheology. Korea-Aust Rheol J 25, 67-75.

Yun H, Oh HJ, Kim MK, Kwak HW, Lee JY, Um IC, Shyam KV, Lee KH (2013) Extraction conditions of Antherea mylitta sericin with high yields and minimum molecular weight degradation. Int J Biol Macromol 52, 59-65. 